\title{
The Hippocampus and Spatial Memory: Findings with a Novel Modification of the Water Maze
}

\author{
Robert E. Clark, ${ }^{1,2}$ Nicola J. Broadbent, ${ }^{2}$ and Larry R. Squire ${ }^{1,2,3,4}$ \\ ${ }^{1}$ Veterans Affairs Medical Center, San Diego, California 92161, and Departments of ${ }^{2}$ Psychiatry, ${ }^{3}$ Neurosciences, and ${ }^{4}$ Psychology, University of California \\ San Diego, La Jolla, California 92093
}

\begin{abstract}
For many tasks and species, remote memory (but not recent memory) is spared after damage to the hippocampus. An exception to this pattern of findings has been that both recent and remote memory are impaired after hippocampal lesions when rats are trained in the conventional water maze task. We explored the effect of introducing a navigational beacon for rats to use during testing. Four identical beacons were hung directly over each of the water maze quadrants, equidistant from each other (multiple-beacon maze). One of the beacons was always directly over the hidden platform. By using distal spatial cues, rats could select the correct beacon and use that beacon as a guide to the hidden platform. Probe tests indicated that rats did use the beacons to guide performance throughout training. Two months after the completion of training, rats were given hippocampal or sham lesions. Controls performed well, but the lesion group performed at chance on the retention probe trials. Furthermore, the rats with lesions not only searched indiscriminately in all four quadrants, they also did not use the beacons. These results indicate that impaired performance in the water maze after hippocampal damage reflects more than a loss of spatial information.
\end{abstract}

Key words: spatial; remote; consolidation; rat; water maze; hippocampus

\section{Introduction}

Studies in humans have documented that damage to the hippocampus produces retrograde amnesia. Typically, the retrograde amnesia is temporally graded, such that information acquired long before hippocampal damage is remembered better than information acquired recently (Kapur and Brooks, 1999; Manns et al., 2003; Squire and Bayley, 2007). Temporally graded retrograde amnesia has been studied extensively in the experimental animal using prospective methods. When the hippocampus is damaged at different times after learning, the large majority of studies have found that remote memory is spared relative to more recent memory (for review, see Squire et al., 2001, 2004; Frankland and Bontempi, 2005).

A notable and consistent exception to this pattern is found in studies with rats that used the benchmark test of spatial memory, the Morris water maze. In the water maze, recent and remote spatial memory were similarly impaired after hippocampal damage (Bolhuis et al., 1994; Mumby et al., 1999; Sutherland et al., 2001; Clark et al., 2005a; Martin et al., 2005). We have performed a series of studies using permanent lesions and reversible lesions and have tested spatial memory in the water maze, a dry-land

Received Jan. 17, 2007; revised May 4, 2007; accepted May 7, 2007.

This work was supported by the Medical Research Service of the Department of Veterans Affairs, the National Institute of Mental Health, the Metropolitan Life Foundation, National Institute on Aging Grant P50 AG05131, the National Science Foundation, the James S. McDonnell Foundation, and a National Alliance for Research on Schizophrenia and Depression Effie Beeman Investigator Award. We thank Laura Entwistle and Daniel Guadarrama for assistance.

Correspondence should be addressed to Dr. Robert E. Clark, Department of Psychiatry, 0603, University of California San Diego, La Jolla, CA 92093. E-mail: reclark@ucsd.edu.

DOI:10.1523/JNEUROSCI.0913-07.2007

Copyright $\odot 2007$ Society for Neuroscience $\quad$ 0270-6474/07/276647-08\$15.00/0 version of the water maze [the Oasis maze (Clark et al., 2005a)], and the annular water maze (Hollup et al., 2001b) to evaluate memory and hippocampal function systematically (Clark et al., 2005a,b; Broadbent et al., 2006). In every instance, both recent and remote memory were equally and robustly impaired. This impairment could reflect the continuing importance of the hippocampus for spatial memory, or the impairment could reflect some performance impairment that is caused by hippocampal damage.

Accordingly, we have considered ways to evaluate the possibility that hippocampal lesions produce some performance/navigational impairment in the water maze task. Here, we introduce a novel modification to the standard water maze that requires rats to abandon a strictly spatial strategy and to use a navigational beacon to guide their search. In this task (the multiple-beacon maze), four identical beacons are suspended directly over the four quadrants of the water maze pool (one per quadrant) (see Fig. 1). One of the beacons is always directly over the hidden platform in the target quadrant. Because the four beacons are identical, the rat must first use spatial knowledge to identify which beacon marks the platform location and then use the beacon to locate the platform. Once the rats have selected the correct beacon, they relinquish navigation in favor of a line-of-sight guidance strategy involving the target beacon. In this way, the task requires spatial memory but not spatial navigation to ultimately locate the hidden platform.

This new task is ideally suited to examine spatial memory in rats with hippocampal damage. Our task uses four beacons, and spatial information is required to identify the correct one. Once the correct beacon is identified, the animal can swim to it without needing to navigate or otherwise track its position in space. Ac- 

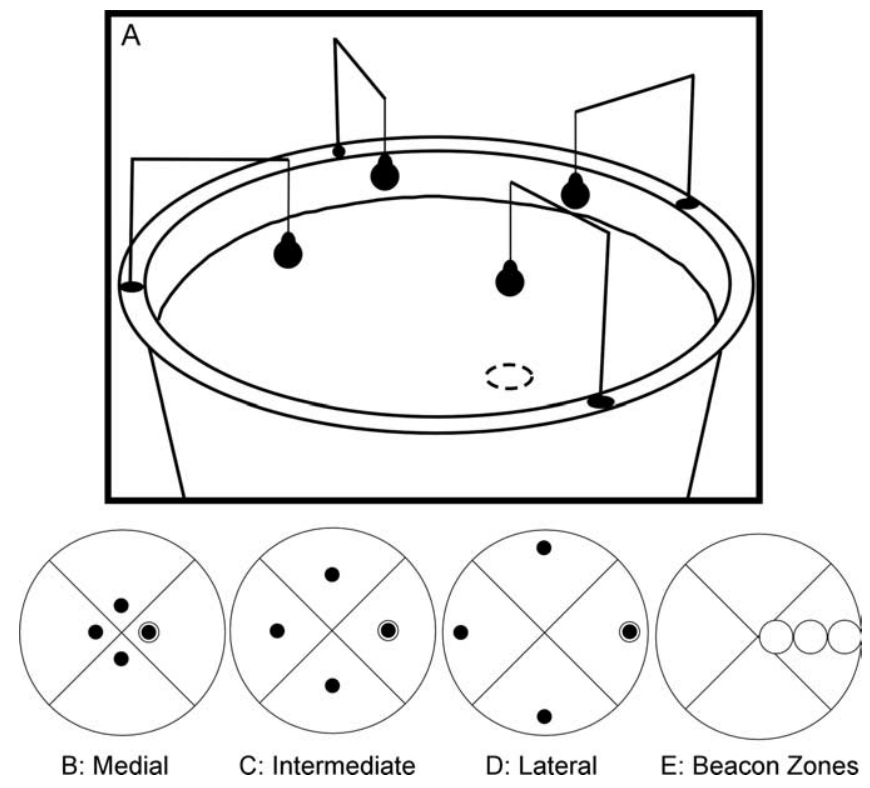

Figure 1. A, Illustration of the water maze pool and the four beacons suspended $25 \mathrm{~cm}$ above the water surface. One of the beacons was always suspended directly over the hidden platform (dashed circle). $\boldsymbol{B}-\boldsymbol{D}$, The hidden platform was always located in the same quadrant of the pool (target quadrant) but could be located in any of three different positions along the center radius of the target quadrant: medial $(\boldsymbol{B})$, intermediate $(\boldsymbol{C})$, and lateral (D). As illustrated, the beacon was always located directly over the hidden platform, and the three other beacons were equidistant from each other (the black circles represent the beacons, and the white circle represents the hidden platform). $\boldsymbol{E}$, To determine whether animals used the beacons during probe trials as guides to the platform location, three equal-sized zones were drawn around the three possible beacon locations within the target quadrant. If animals used the beacons to guide performance, then they should spend more time in the zone marked by the beacon compared with the other two zones in the same quadrant that were not marked by a beacon.

cordingly, the multiple-beacon maze provides the possibility of evaluating spatial memory independently of the ability to navigate through space.

\section{Materials and Methods \\ Subjects}

We tested 16 experimentally naive, male Long-Evans rats. They were $\sim 60 \mathrm{~d}$ old at the beginning of the experiment and weighed $300-320 \mathrm{~g}$. Before surgery, animals were housed in pairs and maintained on a $12 \mathrm{~h}$ light/dark cycle with ad libitum access to food and water.

\section{Apparatus}

Multiple beacon maze. Testing was conducted in a pool of water $(1.6 \mathrm{~m}$ diameter) that was rendered opaque by the addition of powdered milk. The water was maintained at room temperature. The room was illuminated by four $30 \mathrm{~W}$ spotlights pointed at a white ceiling. The testing room contained a number of constant, salient visual cues (posters and equipment). A video camera was mounted on the ceiling directly above the pool and was used in conjunction with a video tracking system (San Diego Instruments, San Diego, CA) to record the swim path of each rat.

We used an Atlantis platform that could be raised or lowered remotely (Spooner et al., 1994). When the platform (12.7 cm diameter) was in the lowered position, the rat could neither detect the platform nor escape from the water. When the platform was in the raised position $(1.5 \mathrm{~cm}$ below the surface of the water), it remained invisible to the rat but provided a means to escape the water.

Multiple beacons. The pool was divided into four quadrants. Four beacons (150 W light bulbs painted flat black that were $7 \mathrm{~cm}$ in diameter) were suspended $25 \mathrm{~cm}$ over the water surface. Each quadrant contained one beacon, and one of the beacons was always suspended directly over the hidden platform (Fig. $1 A$ ). The hidden platform was always located in the same quadrant of the pool but could occupy one of three different positions (medial, intermediate, and lateral) along the center radius of the quadrant (Fig. $1 B-D$ ). The position of the hidden platform was pseudo-randomly changed after each trial, as were the beacons, so that the beacon in the target quadrant was always located directly over the hidden platform and the three other beacons were equidistant from each other and in the other three quadrants. This is the key feature of the task. The beacons are kept relevant by moving them on each trial with the result that the beacons provide unique information that is not available from the distal cues.

\section{Behavioral training}

Rats were given eight trials each day (intertrial interval, $\sim 8 \mathrm{~min}$; two trials per day from each of four starting points and with the sequence of starting points counterbalanced within groups). Testing continued for 5 consecutive days each week for a total of $10 \mathrm{~d}$. Each trial began with the platform in its lowered, inaccessible position. The platform was raised to a submerged but accessible position when the rat dwelled within $20 \mathrm{~cm}$ of the platform for $3 \mathrm{~s}$. After escaping, the rat remained on the platform for $30 \mathrm{~s}$. The first and fifth trials of each training day were $30 \mathrm{~s}$ reinforced probe trials. During these trials, the platform remained in the lowered position and was raised after $30 \mathrm{~s}$. This procedure allowed spatial learning to be tracked across training.

The positions of the hidden platform and the beacons were changed after each trial so that the rats would continue to use the beacon in the target quadrant to guide performance. The requirement that the rat had to dwell over the platform meant that it had to first identify the position of the platform. The platform could be located only when rats used spatial cues to identify the target quadrant and then used the beacon to locate the platform within the target quadrant.

After training, animals were divided into two groups of eight each (control and lesion), equated for preoperative probe trial performance on the first probe trial during the final day of training. Surgery was performed $60 \mathrm{~d}$ after the completion of training.

\section{Surgery and histology}

Anesthesia was maintained throughout surgery with isoflurane gas ( $0.8-$ $2.0 \%$ isoflurane delivered in $\mathrm{O}_{2}$ at $1 \mathrm{~L} / \mathrm{min}$ ). The animal was placed in a David Kopf Instruments (Tujunga, CA) stereotaxic instrument, and the incisor bar was adjusted so that bregma was level with lambda. Thermocoagulation lesions were made with a radiofrequency electrode and generator (model RF-4A; Radionics, Burlington, MA) [see Clark et al. (2000) for additional details]. Lesions were intended to damage the dorsal and ventral hippocampus and were made at multiple locations (all coordinates are in millimeters and relative to bregma): anteroposterior (AP) -2.4 , mediolateral $(\mathrm{ML}) \pm 1.0$, dorsoventral $(\mathrm{DV})-3.5$; $\mathrm{AP}-3.2$, ML $\pm 1.4, \mathrm{DV}-2.7 ; \mathrm{AP}-3.2, \mathrm{ML} \pm 3.0, \mathrm{DV}-2.7 ; \mathrm{AP}-4.0, \mathrm{ML} \pm 2.5, \mathrm{DV}$ -2.3; $\mathrm{AP}-4.0, \mathrm{ML} \pm 3.7, \mathrm{DV}-2.7$; $\mathrm{AP}-4.8, \mathrm{ML} \pm 4.9, \mathrm{DV}-6.8$; $\mathrm{AP}$ $-4.8, \mathrm{ML} \pm 4.3, \mathrm{DV}-7.4,-3.5 ; \mathrm{AP}-5.4, \mathrm{ML} \pm 4.2, \mathrm{DV}-4.2 ; \mathrm{AP}-5.4$, $\mathrm{ML} \pm 5.0, \mathrm{DV}-6.5,-5.5,-4.5$. The wounds were then closed, and the rats recovered from anesthesia on a water-circulating heating pad. Animals were allowed to recover for $14 \mathrm{~d}$ before behavioral testing. Control animals did not receive any surgical procedures.

\section{Postoperative retention probes}

Fourteen days after surgery and $74 \mathrm{~d}$ after the completion of training, three $60 \mathrm{~s}$ nonreinforced probe trials were given with the platform remaining in the inaccessible position. After each probe trial, the rat was removed from the pool. In this way, performance on the probe trials was not confounded by relearning. Each probe trial began with all four beacons in place. The rat was placed in the water facing the pool wall at one of the four start points (counterbalanced within groups). The beacons were located in the intermediate position on the first probe trial (Fig. 1C) and in the lateral (Fig. $1 D$ ) and medial (Fig. $1 B$ ) positions on the second and third probe trials.

Performance on the probe trials was calculated in two ways. First, we measured the percentage of time that each rat spent in the quadrant of the pool where the platform had been located during training (chance, 25\%). Second, to determine whether rats were using the beacons to guide performance, we calculated the amount of time that the rats spent in the area (30 $\mathrm{cm}$ in diameter, $2.8 \%$ of the total pool area) immediately below the beacon (Fig. $1 E$ ). This value was then compared with the amount of time 
spent in the two adjacent areas of equal size in the same quadrant that did not contain the beacon (Fig. $1 E$ ). For example, on the probe trial when the beacons were located in the intermediate position, we compared the time that the rat spent in this intermediate position with the time spent in the medial and lateral positions.

\section{Single-beacon acquisition}

After the three postoperative retention probe trials, both groups of rats were trained on a different version of the task. In this subsequent version of the task, the training occurred in the same water maze, but the hidden platform was moved to the opposite quadrant where it remained in the intermediate position and only one beacon was used. This beacon was suspended directly over the hidden platform and $25 \mathrm{~cm}$ above it. Rats were given $5 \mathrm{~d}$ of training. At the beginning of each day, rats were given a $60 \mathrm{~s}$ reinforced probe trial, followed by four training trials from each of four starting points and with the sequence of starting points counterbalanced within groups.

\section{Histology}

At the completion of behavioral testing, rats were administered an overdose of sodium pentobarbital and perfused transcardially with buffered $0.9 \% \mathrm{NaCl}$ solution, followed by $10 \%$ formaldehyde (in $0.1 \mathrm{M}$ phosphate buffer). The brains were then removed and cryoprotected in $20 \%$ glycerol $/ 10 \%$ formaldehyde. Coronal sections $(50 \mu \mathrm{m})$ were cut with a freezing microtome. Every fifth section was mounted and stained with thionin to assess the extent of the lesions.

\section{Results}

Histological findings

Figure 2 illustrates the extent of the largest and smallest lesion in each group.

\section{Hippocampal damage}

All rats sustained extensive bilateral damage to all the cell fields of the hippocampus and dentate gyrus. The average percentage of damage to the hippocampus was $87.5 \%$ (range, $80.2-94.0 \%$ ). All animals sustained minor damage to the ventral subiculum, and this damage was located in the ventral subiculum just below the ventral hippocampus. All animals also had some damage to the alveus and to the fimbria on the dorsal edge of the dorsal hippocampus. The primary areas of spared hippocampal tissue were located in the most ventromedial portion of the ventral hippocampus and along the midline of the dorsal hippocampus. This sparing involved primarily the most medial aspects of CA1 and the crest of the dentate gyrus. Occasionally, small islands of spared hippocampal tissue were observed within the damaged areas of the hippocampus. The region of the subiculum posterior to the ventral hippocampus, which accounts for $\sim 90 \%$ of subicular tissue, was spared in all cases. The entorhinal cortex was entirely spared in all rats. In addition, none of the animals had damage to the perirhinal cortex or the amygdala.

\section{Other damage}

In all cases, there was detectable damage to the cortical regions directly dorsal to the dorsal hippocampus. In some cases, the damage took the form of cortical thinning, and in the other cases, there was clear damage that was likely attributable to the vascular damage caused by mechanical placement of the probe. Importantly, we detected no damage to the thalamus or to primary visual cortical areas. Thus, there was no anatomical evidence to suggest that the rats in this study had impaired visual function.

\section{Behavioral findings}

\section{Acquisition}

All rats readily learned to identify the target quadrant using spatial cues and also to use the beacons to guide them to the platform location. By the final day of training (first daily probe trial), the

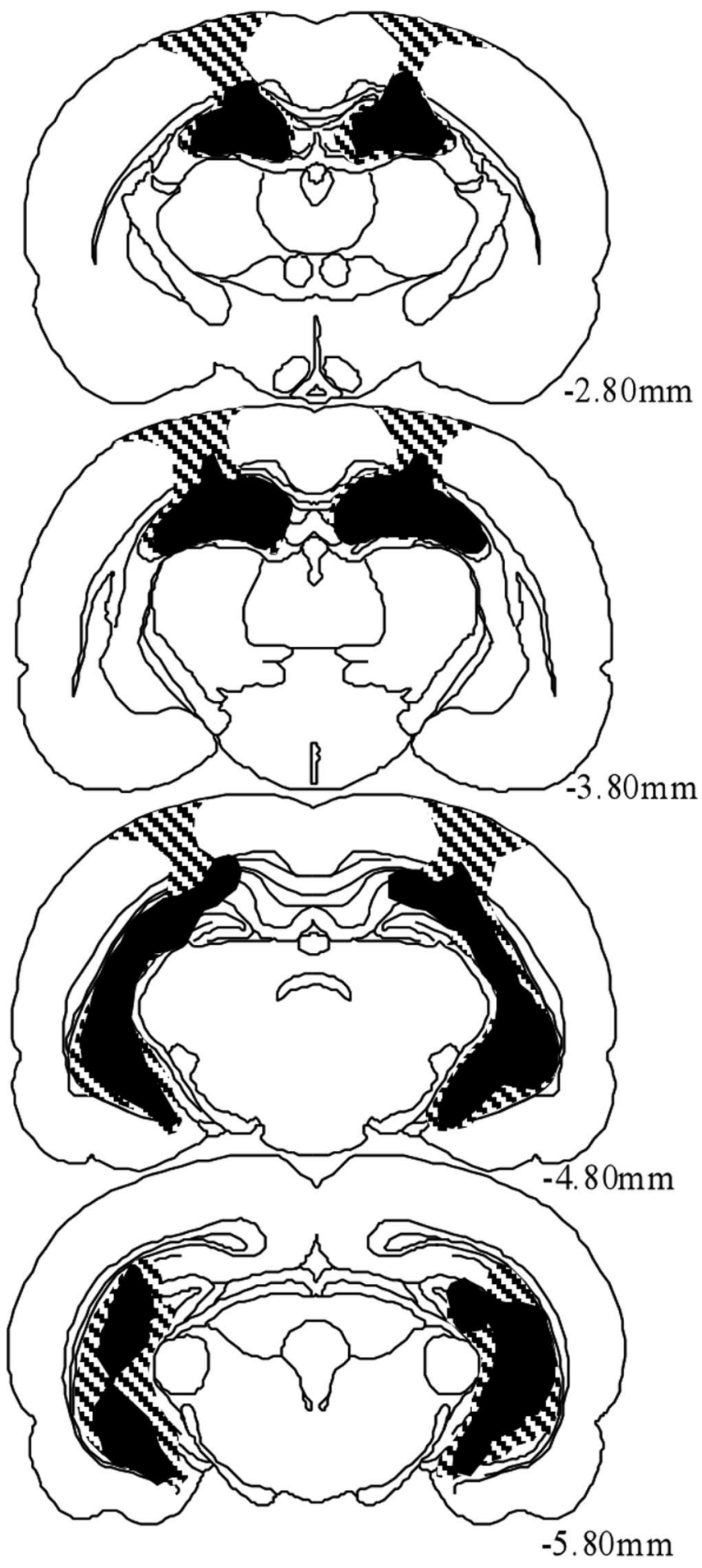

Figure 2. Coronal reconstructions showing the largest (striped) and smallest (black) areas of damage for rats with hippocampal lesions. The series of sections progress (top to bottom) from anterior to posterior levels. Numbers represent the distance in millimeters posterior to bregma.

control group spent $64 \pm 9.3 \%$ of the 30 s probe trial in the target quadrant, and the group that would receive lesions spent nearly the same amount of time $\left(62.2 \pm 8.1 \% ; t_{(14)}=0.2 ; p>0.1\right)$. Figure 3 shows the mean percentage of time during training that each group spent in the target zone beneath the beacon versus the percentage of time each group spent in the other zones that were not beneath the beacon (non-target). Scores were averaged across all 20 probe trials given during the $10 \mathrm{~d}$ of acquisition training. 


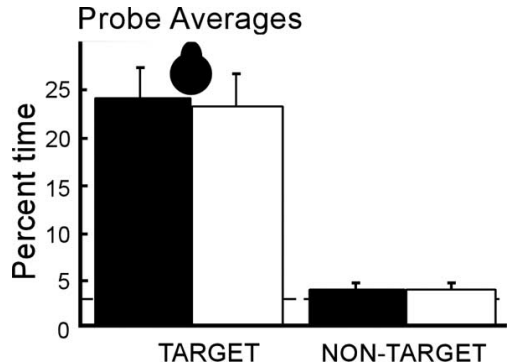

Figure 3. Performance of the control group (filled bars; $n=8$ ) and the to-be-lesioned group (open bars; $n=8$ ) during acquisition training. Bars show the percentage of time, averaged across all 20 probe trials given during $10 \mathrm{~d}$ of acquisition training, that each group spent in the circular zone beneath the beacon (target) versus the percentage of time each group spent in the other two identically sized zones that were not beneath the beacon (non-target). The scores are the percentage of time each group spent in a $30 \mathrm{~cm}$ (diameter) zone directly below the beacon within the target quadrant (chance, $2.8 \%$; dashed line). The beacon icon above the bars identifies the target zones that were under the beacon. Both groups performed the same and spent more time in the target zones than in the non-target zones. Thus, the rats used the beacon in the target quadrant to guide their performance during training. Error bars indicate SEM.

The two groups did not differ from each other in the amount of time they spent in the target and non-target zones (all $t<0.2$; all $p>0.1$ ). Importantly, both groups spent a substantially greater amount of time in the zones beneath the beacon (target) than in the zones that were not beneath the beacon (non-target) (all $t_{(7)}>5.4$; all $\left.p<0.001\right)$. These data indicate that the rats used the beacon in the target quadrant to guide their performance during training.

\section{Acquisition performance compared with standard water maze training}

We were also interested in determining how the beacons affected performance compared with standard water maze training (i.e., in which no beacons were used). In a previous study (Clark et al., 2005a), we trained rats with the exact training parameters used in the present study (eight trials per day for $10 \mathrm{~d}$ with probe trials on the first and fifth trials of each day). Accordingly, we compared performance on the first probe trial of those days when the beacons were located in the intermediate position (which would place the platform in the same location as it was always placed in the standard water maze). In the present study, that condition occurred on the days 3, 4, and 10 of acquisition. The percentage of time in the target quadrant was higher in the multiple-beacon maze than in the standard water maze on all $3 \mathrm{~d}$ (supplemental Fig. 2, available at www.jneurosci.org as supplemental material) and was significantly higher by day 10 (day 3, 52.9 vs $46.8 \%$; day $4,56.8$ vs $49.8 \%$; day $10,64.5$ vs $\left.50.4 \% ; t_{(54)}=2.0 ; p<0.05\right)$. These results indicate that the beacons provided an advantage that was unavailable to rats tested in the standard water maze.

\section{Retention probes: percentage of time in training quadrant}

Figure 4 shows the percentage of time that control rats and rats with hippocampal lesions spent in the training quadrant during the retention probe trials.

Control performance. For the control group, the percentage of time spent in the target quadrant was $45.2 \pm 11.1,61.9 \pm 10.6$, and $56.4 \pm 6.8 \%$ on probe trials $1-3$, respectively (chance, $25 \%$ ). Performance on probe trials 2 and 3 was above chance $\left(t_{(7)}=3.5\right.$, $\left.p<0.05 ; t_{(7)}=4.6, p<0.01\right)$, although not on probe trial 1 $\left(t_{(7)}=1.8 ; p=0.11\right)$. We suggest that the first probe trial might serve as a reminder with respect to the nature of the task. During the first probe trial, the control rats were likely reorienting themselves with the task, and this reorientation or reminder experi-

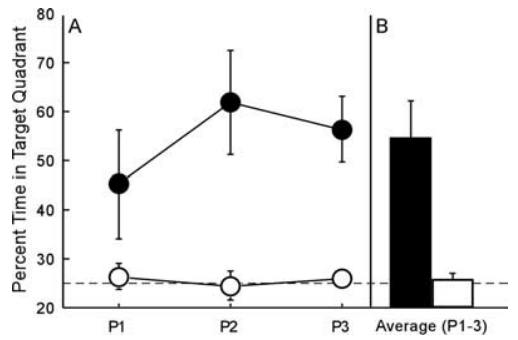

Figure 4. $\boldsymbol{A}$, Performance of the control group (filled circles; $n=8$ ) and the hippocampal lesion group (open circles; $n=8$ ) on three 60 s nonreinforced probe trials given $74 \mathrm{~d}$ after the final day of acquisition training ( $60 \mathrm{~d}$ training-surgery interval). The scores represent the percentage of time each group spent in the target quadrant during each probe trial (chance, 25\%; dashed line). The control group spent marginally more time in the target quadrant than the hippocampal lesion group on probe trial $1(\mathrm{P} 1)$ and significantly more time on probe trials 2 and 3 (P2 and P3, respectively). $\boldsymbol{B}$, Mean performance on the three nonreinforced probe trials for the control group (filled bar) and the lesion group (open bar). The control group performed above chance and better than the lesion group. Error bars indicate SEM.

ence was then manifested as performance that was significantly above chance on probe trial 2. Importantly, there was no opportunity to gather any specific information about the platform location on the first probe trial (because the rats were removed at the end of the probe trial without locating the platform). The average score across all three probe trials was $54.5 \pm 6.8 \%$, a value well above chance $\left(t_{(7)}=3.9 ; p<0.01\right)$. These findings demonstrate that the control group retained the location of the target quadrant.

Lesion group performance. For the lesion group, the percentage of time spent in the target quadrant was $26.3 \pm 2.7,24.5 \pm 2.9$, and $25.9 \pm 1.3 \%$ on probe trials $1-3$, respectively. None of these scores were above chance (all $t<0.8$; all $p>0.1$ ). There was not a reliable difference between the lesion group and the control group on probe trial $1\left(t_{(14)}=1.7 ; p=0.11\right)$, but the lesion group was impaired relative to the control group on probe trials 2 and 3 as well as when performance across all three probe trials was compared (all $t>3.4$; all $p<0.01$ ).

\section{Retention probes: percentage of time near the beacons}

We next determined to what extent the groups used the beacons to guide performance within the target quadrant. For each probe trial, we drew a $30-\mathrm{cm}$-diameter circle in the target quadrant beneath the location of the beacon and compared the percentage of time that each group spent in that area to the percentage of time each group spent in the two other equal-sized zones within the target quadrant that did not contain a beacon (Fig. $1 E$ ). If animals were using the beacons on the probe trials, they should have spent a disproportionate amount of time in the zone that contained the beacon.

Probe 1 (intermediate beacon). The control group spent marginally more time in the intermediate beacon-containing zone than would be expected by chance (14.8 $\pm 5.4 \%$; chance, $2.8 \%$; $\left.t_{(7)}=2.2 ; p=0.06\right)$, significantly more time in the intermediate (beacon-containing) zone than in the lateral zone $\left(t_{(7)}=2.5 ; p<\right.$ 0.05 ) (Fig. 5A). In contrast, the lesion group did not discriminate among the three zones and did not spend more time in any of the zones than would be expected by chance (all $t<1.0$; all $p>0.1$ ).

Probe 2 (lateral beacon). The control group spent more time in the lateral beacon-containing zone than would be expected by chance $\left(19.6 \pm 4.9 \%\right.$; chance, $\left.2.8 \% ; t_{(7)}=3.4 ; p<0.05\right)$ and significantly more time in the lateral zone than in the medial zone $\left(t_{(7)}=2.5 ; p<0.05\right.$ ) (Fig. $5 B$ ). In contrast, the lesion group did not discriminate among the three zones and spent no more time 

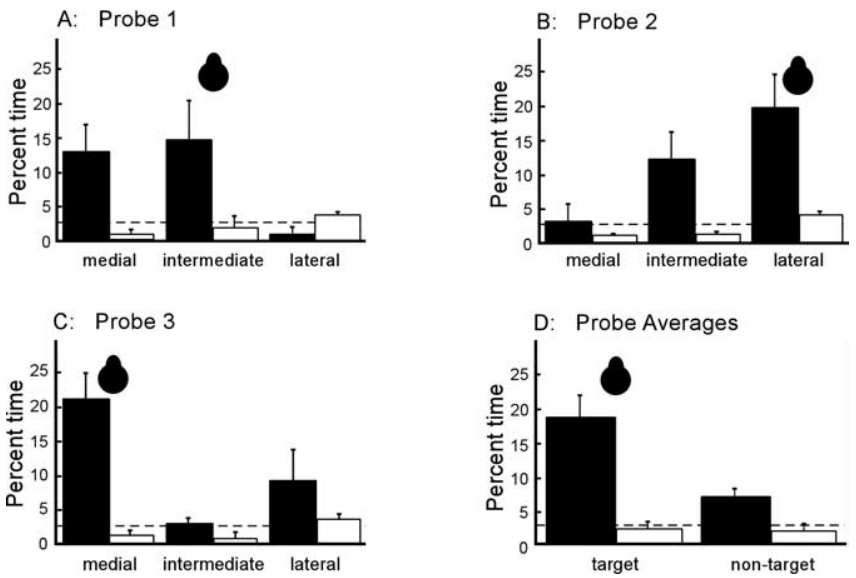

Figure 5. $A-C$, Performance of the control group (filled bars; $n=8$ ) and the lesion group (open bars; $n=8$ ) on each of three nonreinforced probe trials when the beacons were located in the intermediate position $(\boldsymbol{A})$, the lateral position $(\boldsymbol{B})$, and the medial position $(\boldsymbol{C})$ within the target quadrant. The beacon icon above the bars identifies the zone that was beneath the beacon. The scores are the percentage of time each group spent in a $30 \mathrm{~cm}$ (diameter) zone directly below the beacon within the target quadrant (chance, $2.8 \%$; dashed line). $\boldsymbol{D}$, Percentage of time (averaged across the three probe trials) spent by each group in the circular zone beneath the beacon (target) versus the percentage of time spent in the other two circular zones that did not contain the beacon (non-target). Within the target quadrant, control animals spent more time in the zone marked by a beacon than in the other two zones not marked by a beacon. Error bars indicate SEM.

in any of the zones than would be expected by chance (all $t<1.0$; all $p>0.1)$.

Probe 3 (medial beacon). The control group spent more time in the medial, beacon-containing zone than would be expected by chance $\left(21.2 \pm 4.0 \%\right.$; chance, $\left.2.8 \% ; t_{(7)}=4.6 ; p<0.01\right)$ and more time in the medial zone than in the intermediate zone $\left(t_{(7)}=4.6 ; p<0.01\right.$ ) (Fig. $5 C$ ). In contrast, the lesion group did not discriminate among the three zones and spent no more time in any of the zones than would be expect by chance (all $t<1.0$; all $p>0.1)$.

Average of three probe trials (beacon vs non-beacon zones). We next compared the percentage of time across all three probe trials that the two groups spent in the zones beneath the beacon with the percentage of time that the two groups spent in the zones that were not beneath the beacon (Fig. 5D). The control group spent more time in the beacon-containing zones than in the other zones $\left(18.5 \pm 3.5\right.$ vs $\left.7.0 \pm 1.1 \% ; t_{(7)}=4.6 ; p<0.01\right)$, whereas the lesion group did not discriminate among the beacon and nonbeacon zones (all $p>0.1$ ).

These results indicate that the animals in the control group used the beacon within the target quadrant to guide and focus their search. In contrast, the lesion group exhibited no tendency to use the beacons. Importantly, the lesion group also did not tend to search beneath the beacons in the non-target quadrants (time in beacon-containing zones in the three non-target quadrants vs time in non-beacon zones in those quadrants; all $t<1.0$; all $p>0.1$ ). Thus, the lesion group not only failed to exhibit a preference for the target quadrant, they did not use the beacons to guide their search. Figure 6 shows where the control and lesion groups distributed their searches during the three probe trials. The position of each rat was sampled every $200 \mathrm{~ms}$, and the 300 positions occupied during each of the three $60 \mathrm{~s}$ probe trials are indicated with a black dot. These plots show the positions occupied by every rat in the control group (left column) and every rat in the lesion group (right column) for probe trial 1 (first row), probe trial 2 (second row), and probe trial 3 (third row). The
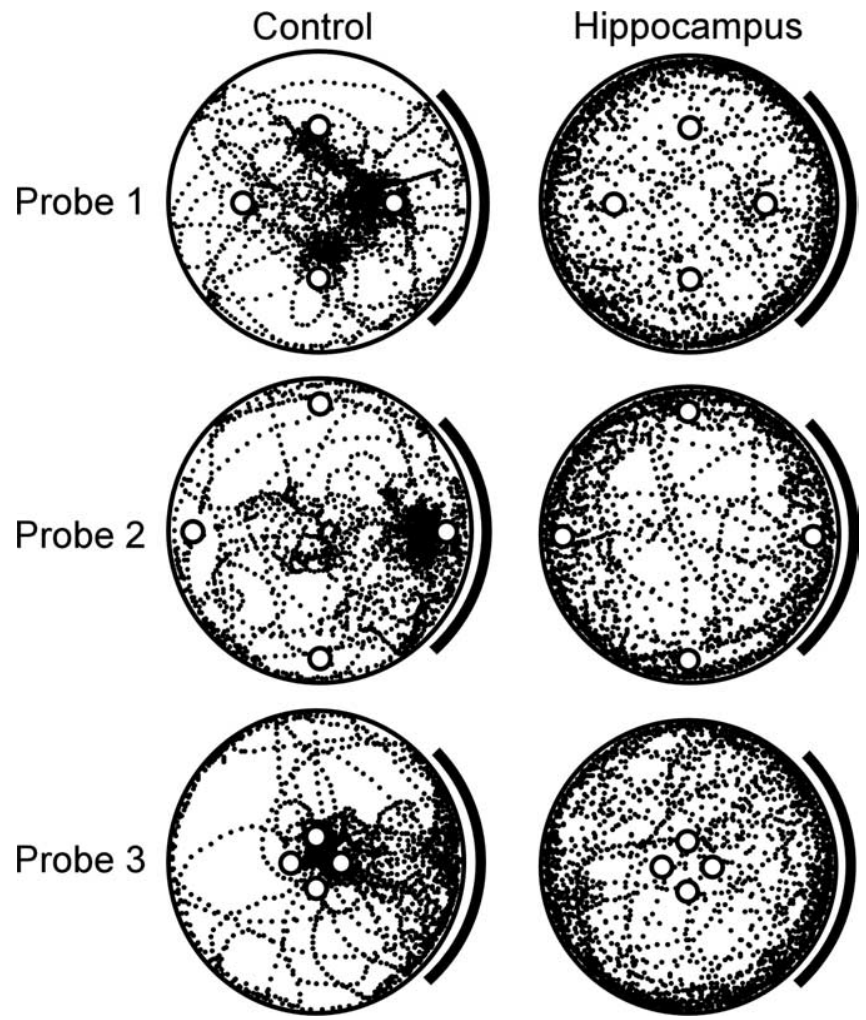

Figure 6. The position of each rat was sampled every $200 \mathrm{~ms}$ during each 60 s probe trial and indicated with a black dot. The plots thus show the 300 positions occupied by every rat in the control group (left column) and every rat in the lesion group (right column) during probe trial 1 (first row), probe trial 2 (second row), and probe trial 3 (third row). The white circles show the position of the beacons on each probe trial. The target quadrant is on the east side of each circle as indicated by the $90^{\circ}$ black arc. Note that the animals in the control group spent a disproportionate amount of time in the target (east) quadrant in a focused search near the appropriate beacon. The animals in the lesion group did not focus their search in any particular quadrant and also failed to use the beacons to focus their search.

control group consistently searched under the beacon within the target quadrant. In sharp contrast, the lesion group did not follow the beacon. Instead, the animals in the lesion group distributed their search around the edge of the pool. This search strategy is termed thigmotaxis and is characteristic of naive rats when they are first placed in the water maze.

To further evaluate the search strategies of the two groups, we also compared the search paths on the first probe trial of acquisition (i.e., the first time the rats were placed in the pool) with the swim paths on the first postoperative probe trial. Thus, we compared the search paths of animals when they were completely naive with the search paths of the same animals after they had $10 \mathrm{~d}$ of spatial training and (60 d later) sham lesions or hippocampal lesions. Because naive rats spend a disproportionate amount of time searching the perimeter of the pool (thigmotaxis), we drew a zone around the perimeter of the pool to create an annulus that encompassed $33 \%$ of the total surface area of the pool. We then compared the percentage of time each group spent in this annulus on the first probe trial of acquisition and on the first postoperative probe trial. When naive, both groups spent an equal amount of time in the peripheral zone (control, $79.2 \pm 2.3 \%$; preoperative $\left.\mathrm{H}-\mathrm{RF}, 83.5 \pm 3.8 \% ; t_{(14)}=1.0 ; p>0.1\right)$ and more time than would be predicted by chance (all $t_{(7)}>13.0$; all $p<$ $0.0001)$. On the first postoperative probe trial, the control group spent only $12.6 \pm 3.3 \%$ of the time in the peripheral zone, considerably less than when they were naive $\left(t_{(7)}=15.1 ; p<0.01\right)$. In 
contrast, on the first postoperative probe trial, the lesion group spent $75.6 \pm 6.1 \%$ of the time in the peripheral zone, about the same amount of time as on the first acquisition probe trial at the beginning of training $\left(t_{(7)}=1.2 ; p>0.1\right)$. These results indicate that the animals in the lesion group not only failed to exhibit spatial memory retention and failed to the use beacons to guide their performance, they also failed to retain any search strategies that would distinguish them from a completely naive animal.

\section{Single-beacon acquisition}

We measured the latency (in seconds) each rat took to reach the platform location on the five trials each day and calculated an average daily latency for both groups across the $5 \mathrm{~d}$ of training. Supplemental Figure 1 (available at www.jneurosci.org as supplemental material) shows the average daily latency for the two groups. A repeated-measures ANOVA indicated a reduction in daily latency across the $5 \mathrm{~d}$ of testing for both the control group $\left(F_{(4,28)}=51.2 ; p<0.0001\right)$ and the lesion group $\left(F_{(4,28)}=3.6\right.$; $p<0.02)$. The lesion group exhibited longer latencies than the control group across each of the five testing sessions (all $t>2.4$; all $p<0.02$ ).

These data show that although the lesioned group remained impaired relative to the control group, the lesion group had sufficient sensory abilities to acquire some of the procedural aspects of this task. The longer latencies likely reflect the fact that this task differs from the conventional visible platform task and that some hippocampal function is needed to learn that the beacon is an indicator of platform location.

\section{Discussion}

Using a novel multiple-beacon maze, we found that rats can readily use distal spatial cues to identify a target quadrant and use the target beacon to guide them to a hidden platform. Because the hidden platform could be in one of three different positions within the target quadrant, the rats had to continue to use the target beacon across $10 \mathrm{~d}$ of training. Figure 3 illustrates that both groups of animals did, in fact, use the target beacon during training, and supplemental Figure 2 (available at www.jneurosci.org as supplemental material) illustrates that the presence of the beacons improved performance over what occurs when rats are trained in the standard version of the water maze.

After the 10 week retention interval, the control group exhibited retention of the spatial location of the target quadrant. Controls performed marginally above chance on the first probe trial, greater than chance on probe trials 2 and 3, and greater than chance across all three nonreinforced probe trials. Because the probe trials were nonreinforced (i.e., the platform was never available), these trials represent a measure of retention uncomplicated by the possibility of relearning. Figure 5 shows that as the beacons were repositioned on each of the three probe trials, the control group consistently spent more time in the beaconcontaining zone compared with the adjacent non-beacon zones. Thus, during the retention test, the rats in the control group used the beacon within the target quadrant to guide their search for the platform. Furthermore, one can see in Figure 6 that the rats searched the target beacon and also searched in the direction of the two adjacent beacons (and to a lesser extent, the opposite beacon). A similar pull toward the adjacent beacons was apparent for probe 3 . For probe 2 , it seems likely that the rats were not pulled by the adjacent beacons because they were too distant from the target beacon. Thus, one can see, in the swim paths of the control animals, that the beacons were guiding behavior.

The results from the lesion group were unambiguous. Specif- ically, the animals in the lesion group performed at chance on all three retention probe trials, despite the fact that the beacons were available to help guide their search. Furthermore, these animals did not use any of the beacons to guide their search. That is, across the three probe trials, these animals never spent more time in beacon-containing zones than in adjacent zones that did not contain a beacon. Because rats with hippocampal lesions can effectively use a single beacon to guide their search to a hidden platform (Morris et al., 1982, 1986; Save and Poucet, 2000), one might have expected that rats trained to use the beacons would retain this ability after hippocampal lesions.

These findings have implications for the interpretation of impaired retention performance of rats in the water maze after hippocampal lesions. Thus, one possible explanation of the poor performance in previous studies has been that animals were searching for an escape platform but could not remember its location. The present results raise instead the possibility that animals have forgotten that there is a platform to find. That is, if hippocampal lesions simply abolished spatial memory of the platform location, one would suppose that rats would have continued to use the beacons to guide their search but would have searched indiscriminately under all the beacons. This result was not obtained. Figure 6 shows that rats in the lesion group exhibited a strong thigmotaxic search pattern with only occasional forays toward more central areas of the pool. In fact, it was not possible to distinguish between the search paths of entirely naive rats and the search paths of these same rats after hippocampal lesions and extended water maze training. The loss of search strategies that we observed after large hippocampal lesions contrasts with the results obtained after infusion of an AMPA/kainite receptor antagonist into the dorsal hippocampus. In that case, spatial memory was impaired, but the acquired search strategy was preserved (Micheau et al., 2004).

It is interesting to note that work with the annular water maze attempted to reduce the navigational demands of the water maze task (Hollup et al., 2001b; Clark et al., 2005a). Nonetheless, evidence is lacking that rats, in fact, approach the annular version of the water maze differently than they approach the standard version of the water maze. That is, rats may use a navigational strategy, although it is not required. In contrast, in the present study, once the rats select the correct beacon, they must relinquish a navigation strategy in favor of a line-of-sight guidance strategy involving the target beacon. The finding that performance remained severely impaired indicated that rats with lesions not only failed to exhibit spatial memory, they also failed to use the beacons.

Our findings therefore indicate that impaired performance after hippocampal lesions on spatial tests like the water maze must involve more than a simple loss of spatial information. One possibility is that hippocampal lesions produced amnesia for all aspects of the task (including the fact that escape is possible within the interior of the pool and that the beacons are relevant) and that this deficit would appear as a spatial impairment when training is conducted in the conventional water maze. Alternatively, the impairment could reflect a loss of memory together with an impaired ability to efficiently execute the requirements of the task. It is also possible that the impairment is attributable entirely to an inability to express an otherwise preserved spatial memory (i.e., an impaired ability to execute the requirements of the retention test). For example, performance impairment could reflect an inability to remain oriented during the retention test or an inability to update spatial position during the course of the retention test (Knowlton and Fanselow, 1998). Such impair- 
ments could be attributable either to impaired anterograde memory or to a loss of the capacity for spatial computation that normally depends on the hippocampus. Finally, it is also possible that the lesion group simply disregarded the beacons because the failure to solve the spatial component of the task made the beacons irrelevant.

Recent electrophysiological evidence suggests that hippocampal cells, in addition to signaling where an animal is in space (i.e., when they behave as place cells), also show prospective memory coding by signaling the turn that the animal is about to make (Ferbinteanu and Shapiro, 2003) as well as the time the rat reaches the goal location (Hok et al., 2007). For example, it has been shown in the annular maze that place fields accumulate in the target location but not in other equally sized areas (Hollup et al., 2001a). Hippocampal cells might therefore participate in a network that allows the rat to plan accurate trajectories in space and ascertain when they have arrived at the correct destination. From this perspective, hippocampal lesions would be expected to affect performance broadly in our task.

It is notable that there is abundant evidence for the idea that spatial memory gradually becomes independent of the hippocampus. The evidence comes from four sources: (1) studies of less demanding spatial memory tasks than the water maze (including context fear conditioning); (2) studies using activitydependent brain imaging; (3) studies with reversible lesions; and (4) studies after selective lesions of hippocampal circuitry. A detailed discussion of this evidence is available at www.jneurosci.org as supplemental material.

A recent study (Teixeira et al., 2006) is particularly relevant to the present work because it involved the water maze in conjunction with imaging of immediate-early genes. First, c-fos expression was more elevated in the anterior cingulate cortex during retrieval of a 1-month-old spatial memory than during retrieval of a 1-d-old spatial memory. Most relevant, c-fos expression in the hippocampus was equally elevated during retrieval of recent and remote memory, both when animals were trained on a spatial version of the task and when animals simply had learned to swim to a visible platform located in variable positions. These findings indicate that the hippocampus was always active during testing, regardless of whether the memory being tested was recent or remote and even when spatial memory was not relevant (i.e., during test trials involving the visible platform). The authors interpreted these results to mean that the hippocampus has a performance/navigation role during the test trials (as well as a possible mnemonic role) (Teixeira et al., 2006).

Furthermore, reversible lesions of the hippocampus impaired both recent and remote spatial memory (Broadbent et al., 2006; Teixeira et al., 2006). Yet reversible lesions of the anterior cingulate cortex impaired remote spatial memory (at 1 month) but not recent spatial memory (at $1 \mathrm{~d}$ ) (Teixeira et al., 2006). The findings for the anterior cingulate cortex imply that even in tasks like the water maze, spatial memory is reorganized over time. Perhaps the results for the water maze differ from the results for other tasks, not because water maze memory does not reorganize (or consolidate) as recent memory becomes remote memory, but because the hippocampus is always essential for performance of the task.

\section{Conclusion}

The critical finding from the present study is that hippocampal lesions produced behavioral impairments that extended beyond the domain of spatial memory. Accordingly, the impairments that are consistently observed in the water maze task after large hippocampal lesions need not be taken to mean that remote spatial memory is formed and permanently stored within the hippocampus or that spatial memory is fundamentally different from other kinds of memory. Instead, the evidence reviewed here suggests that spatial memory reorganizes after training like other nonspatial forms of memory and that (as with other forms of memory) the neocortex becomes gradually more important as time passes. It will be useful to pursue these issues with additional studies using pharmacological and/or genetic methods to disrupt the hippocampus reversibly during the retention interval so that spatial memory can be tested at a time when the hippocampus is "on-line" and able to support the expression of memory (Riedel et al., 1999; Cui et al., 2004; Micheau et al., 2004). Other fruitful lines of research include the use of more selective lesions (Nakazawa et al., 2002; Steffenach et al., 2002; Remondes and Schuman, 2004) and spatial tasks that do not require substantial performance demands for their expression [e.g., the "village" task (Winocur et al., 2005) and the paired-associate "event arena" (Day et al., 2003)].

\section{References}

Bolhuis J, Stewart CA, Forrest EM (1994) Retrograde amnesia and memory reactivation in rats with ibotenate lesions to the hippocampus or subiculum. Q J Exp Psychol 47:129-150.

Broadbent NJ, Squire LR, Clark RE (2006) Reversible hippocampal lesions disrupt water maze performance during both recent and remote memory tests. Learn Mem 13:187-191.

Clark RE, Zola SM, Squire LR (2000) Impaired recognition memory in rats after damage to the hippocampus. J Neurosci 20:8853-8860.

Clark RE, Broadbent NJ, Squire LR (2005a) Hippocampus and remote spatial memory in rats. Hippocampus 15:260-272.

Clark RE, Broadbent NJ, Squire LR (2005b) Impaired remote spatial memory after hippocampal lesions despite extensive training beginning early in life. Hippocampus 15:340-346.

Cui Z, Wang H, Tan Y, Zaia KA, Zhang S, Tsien JZ (2004) Inducible and reversible NR1 knockout reveals crucial role of the NMDA receptor in preserving remote memories in the brain. Neuron 41:781-793.

Day M. Langston R, Morris RGM (2003) Glutamate-receptor-mediated encoding and retrieval of paired-associate learning. Nature 424:205-209.

Ferbinteanu J, Shapiro ML (2003) Prospective and retrospective memory coding in the hippocampus. Neuron 40:1227-1239.

Frankland PW, Bontempi B (2005) The organization of recent and remote memories. Nat Rev Neurosci 6:119-130.

Hok V, Lenck-Santini PP, Roux S, Save E, Muller RU, Poucet B (2007) Goalrelated activity in hippocampal place cells. J Neurosci 27:472-482.

Hollup SA, Molden S, Donnett JG, Moser MB, Moser EI. (2001a) Accumulation of hippocampal place fields at the goal location in an annular watermaze task. J Neurosci 21:1635-1644.

Hollup SA, Kjelstrup KG, Hoff J, Moser M-B, Moser E (2001b) Impaired recognition of the goal location during spatial navigation in rats with hippocampal lesions. J Neurosci 21:4505-4513.

Kapur N, Brooks DJ (1999) Temporally-specific retrograde amnesia in two cases of discrete bilateral hippocampal pathology. Hippocampus 9:247-254.

Knowlton BJ, Fanselow MS (1998) The hippocampus, consolidation and on-line memory. Curr Opin Neurobiol 8:293-296.

Manns JR, Hopkins RO, Reed JM, Kitchener EG, Squire LR (2003) Recognition memory and the human hippocampus. Neuron 37:1-20.

Martin SJ, de Hoz L, Morris RG (2005) Retrograde amnesia: neither partial nor complete hippocampal lesions in rats result in preferential sparing of remote spatial memory, even after reminding. Neuropsychologia 43:609-624.

Micheau J, Riedel G, Roloff EL, Inglis J, Morris RG (2004) Reversible hippocampal inactivation partially dissociates how and where to search in the water maze. Behav Neurosci 8:1022-1032.

Morris RG, Garrud P, Rawlins JN, O’Keefe J (1982) Place navigation impaired in rats with hippocampal lesions. Nature 297:681-683.

Morris RG, Hagan JJ, Rawlins JN (1986) Allocentric spatial learning by hippocampectomised rats: a further test of the "spatial mapping" and "work- 
ing memory" theories of hippocampal function. Q J Exp Psychol B 38:365-395.

Mumby DG, Astur RS, Weisend MP, Sutherland RJ (1999) Retrograde amnesia and selective damage to the hippocampal formation: memory for places and object discriminations. Behav Brain Res 106:97-107.

Nakazawa K, Quirk MC, Chitwood RA, Watanabe M, Yeckel MF, Sun LD, Kato A, Carr CA, Johnston D, Wilson MA, Tonegawa S (2002) Requirement for hippocampal CA3 NMDA receptors in associative memory recall. Science 297:211-218.

Remondes M, Schuman EM (2004) Role for a cortical input to hippocampal area $\mathrm{CAl}$ in the consolidation of a long-term memory. Nature 431:699-703.

Riedel G, Micheau J, Lam AG, Roloff E, Martin SJ, Bridge H, de Hoz L, Poeschel B, McCulloch J, Morris RGM (1999) Reversible neural inactivation reveals hippocampal participation in several memory processes. Nat Neurosci 2:898-905.

Save E, Poucet B (2000) Involvement of the hippocampus and associative parietal cortex in the use of proximal and distal landmarks for navigation. Behav Brain Res 109:195-206.

Spooner RIW, Thomson A, Hall J, Morris RGM, Salter SH (1994) The Atlantis platform: a new design and further developments of Buresova's on-demand platform for the water maze. Learn Mem 3:203-211.
Squire LR, Bayley PJ (2007) The neuroscience of remote memory. Curr Opin Neurobiol 17:185-196.

Squire LR, Clark RE, Knowlton BJ (2001) Retrograde amnesia. Hippocampus 11:50-55.

Squire LR, Clark RE, Bayley PJ (2004) Medial temporal lobe function and memory. In: The cognitive neurosciences, Ed 3 (Gazzinaga M, ed), pp 691-708.

Steffenach HA, Sloviter RS, Moser EI, Moser MB (2002) Impaired retention of spatial memory after transection of longitudinally oriented axons of hippocampal CA3 pyramidal cells. Proc Natl Acad Sci USA 99:3194-3198.

Sutherland RJ, Weisend MP, Mumby D, Astur RS, Hanlon FM, Koerner A, Thomas MJ, Wu Y, Moses SN, Cole C, Hamilton DA, Hoesing JM (2001) Retrograde amnesia after hippocampal damage: recent vs. remote memories in three tasks. Hippocampus 11:27-42.

Teixeira CM, Pomedli SR, Maei HR, Kee N, Frankland PW (2006) Involvement of the anterior cingulate cortex in the expression of remote spatial memory. J Neurosci 26:7555-7564.

Winocur G, Moscovitch M, Fogel S, Rosenbaum RS, Sekeres M (2005) Preserved spatial memory after hippocampal lesions: effects of extensive experience in a complex environment. Nat Neurosci 8:273-275. 